\title{
HIPPOCAMPAL DNA METHYLATION, DNAm AGE AND SPATIAL MEMORY PERFORMANCE IN YOUNG AND OLD RATS
}

Priscila Chiavellini ${ }^{1^{*}}$, Marianne Lehmann ${ }^{1^{*}}$, Martina Canatelli Mallat ${ }^{1}$, Joseph A. Zoller $^{2}$, Claudia B. Herenu ${ }^{3}$, Gustavo R. Morel ${ }^{1}$, Steve Horvath ${ }^{2,4}$, Rodolfo G. Goya ${ }^{1}$

${ }^{1}$ Institute for Biochemical Research (INIBIOLP) - Histology B \& Pathology B, School of Medicine, National University of La Plata (UNLP), La Plata, Argentina

2 Department of Biostatistics, Fielding School of Public Health, University of California, Los Angeles, Los Angeles, California, USA

3 Institute for Experimental Pharmacology (IFEC), School of Chemical Sciences, National University of Cordoba, Cordoba, Argentina

4 Department of Human Genetics, David Geffen School of Medicine, University of California, Los Angeles, Los Angeles, California, USA;

Send correspondence to:

\author{
INIBIOLP \\ Faculty of Medicine, UNLP \\ CC 455 \\ 1900 La Plata \\ Argentina \\ tel: (54-221) 425-6735 \\ fax: (54-221) 425-0924/425-8988 \\ e-mail: goya@isis.unlp.edu.ar
}

Keywords: Aging - Hippocampus- Spatial memory - Methylation landscape - DNAm

age - Regression-Barnes maze - Rat

\footnotetext{
* These two authors contributed equally to this study.
} 


\begin{abstract}
In humans and rats, aging is associated with a progressive deterioration of spatial learning and memory. These functional alterations are correlated with morphological and molecular changes in the brain, particularly in the hippocampus. Here, we assessed the age-related changes in the DNA methylation (DNAm) landscape in the rat hippocampus and assessed the correlation of spatial memory performance with hippocampal DNAm age in young (2.6 mo.) and old (26.6 mo.) rats.

Spatial memory performance was assessed with a modified version of the Barnes maze test. In order to evaluate learning ability as well as spatial memory retention, we assessed the time spent (permanence) by animals in goal sector $1\left(\mathrm{GS}_{1}\right)$ and $3\left(\mathrm{GS}_{3}\right)$ when the escape box was removed. The rat pan-tissue clock was applied to DNA methylation profiles of hippocampal tissue. The bisulfite converted genomic DNA was analyzed by Illumina Infinium HorvathMammalMethylChip40. The Horvath Mammal Methyl Chip40 assay provides quantitative measurements of DNA methylation for $22528 \mathrm{CpG}$ dinucleotides that map to the Rattus norvegicus UCSC 6.0 genome. An enrichment pathway analysis revealed that neuron fate commitment, brain development, and central nervous system development were processes whose underlying genes were enriched in positively methylated CpGs. In the old rat hippocampi, the methylation levels of CpGs proximal to transcription factors associated with genes Pax5, Lbx1, Nr2f2, Hnf1b, Zic1, Zic4, Hoxd9; Hoxd10, Gli3, Gsx1 and Lmx1b, and Nipbl showed a significant regression with spatial memory performance. Regression analysis of different memory performance indices with hippocampal DNAm age was significant when data from young and old rats were taken together. The above results suggest that age-related hypermethylation of certain gene families, like Zic and Gli, may play a causal role in the decline in spatial memory in old rats. Hippocampal DNAm age seems to be a reliable index of spatial memory performance in young and old rats.
\end{abstract}




\section{INTRODUCTION}

In humans and rats, aging is associated with a progressive deterioration of spatial learning and memory. These functional alterations are correlated with morphological changes in the brain, particularly in the hippocampus, a key brain region for the formation and consolidation of spatial memory (1-3). At molecular level, gene expression studies in aging rodents have documented significant changes in hippocampal genes related to cholesterol synthesis, inflammation, transcription factors, neurogenesis and synaptic plasticity (4-8). In the hippocampus of female rats, it was reported that 210 genes are differentially expressed in senile as compared with young counterparts, most of them being downregulated. RNA-Seq data showed that various genes involved in the immune response, including TYROBP, CD11b, C3, CD18, CD4 and CD74, are overexpressed in the hippocampus of aged animals (9).

A highly relevant molecular variable that has not been systematically assessed in the hippocampus of young and old rats is DNA methylation (DNAm). In particular, there are, to our knowledge, no documented studies on the impact of age changes in hippocampal DNA methylation on memory performance. Early ground-breaking studies performed in rat brain showed a global loss of DNA methylation during aging (10) but there is scanty information on the DNAm landscape in the rat hippocampus and even less about the impact of aging on this landscape.

The demonstration that the DNA methylation profile of a specific set of cytosineguanine sites ( $\mathrm{CpG}$ sites) is a highly accurate biomarker of biological age gave rise to the algorithms known as epigenetic clocks (11-16). Since epigenetic clocks seem to reflect biological age (DNAm age), we were interested in correlating a set of variables used to assess spatial memory, with hippocampal DNAm age in young and old rats. The results of the study are reported here.

\section{EXPERIMENTAL PROCEDURES}

\section{Animals}

Thirteen young (average age 2.6 mo.; range \pm 2 days) and 11 old (26.6 mo; range \pm 5 days.) female Sprague-Dawley (SD) rats weighing (X \pm SEM) $165 \pm 5$ and $250 \pm 9 \mathrm{~g}$, respectively, were used. Animals were housed in a temperature-controlled room $(22 \pm$ $2^{\circ} \mathrm{C}$ ) on a $12: 12 \mathrm{~h}$ light/dark cycle. Food and water were available ad libitum. All experiments with animals were performed in accordance with the Animal Welfare Guidelines of NIH (INIBIOLP's Animal Welfare Assurance No A5647-01). The 
ethical acceptability of the animal protocols used here have been approved by our institutional IACUC (Protocol \# T09-01-2013).

\section{Spatial memory assessment}

Description of the Barnes maze protocol used- The modified Barnes maze protocol used in this study was based on a previously reported procedure (3). It consists of an elevated (108 cm to the floor) black acrylic circular platform, $122 \mathrm{~cm}$ in diameter, containing twenty holes around the periphery. The holes are of uniform diameter (10 $\mathrm{cm}$ ) and appearance, but only one hole is connected to a black escape box (tunnel). The escape box is $38.7 \mathrm{~cm}$ long $\times 12.1 \mathrm{~cm}$ wide $\times 14.2 \mathrm{~cm}$ in depth and it is removable. A white squared starting chamber (an opaque, $20 \mathrm{~cm}$ x $30 \mathrm{~cm}$ long, and $15 \mathrm{~cm}$ high, openended chamber) was used to place the rats on the platform. Four proximal visual cues were placed in the room, $50 \mathrm{~cm}$ away from the circular platform. The escape hole was numbered as hole 0 for graphical normalized representation purposes, the remaining holes being numbered 1 to 10 clockwise, and -1 to -9 counterclockwise (Fig. 5-Panel A). Hole 0 remains in a fixed position, relative to the cues in order to avoid randomization of the relative position of the escape box. During the tests the platform was rotated daily. A 90-dB white-noise generator and a white-light $500 \mathrm{~W}$ bulb provided the escape stimulus from the platform. We used an abbreviated protocol based on three days of acquisition trials (AT), followed by a probe trial (PT) (one day after training) to assess spatial memory retention. An AT consists of placing a rat in the starting chamber for $30 \mathrm{~s}$, the chamber is then raised, and the aversive stimuli (bright light and high pitch noise) are switched on and the rat is allowed to freely explore the maze for $120 \mathrm{~s}$. A probe trial is defined as a trial where the escape box has been removed, its purpose being to assess the exploration frequency of the empty escape hole and nearby holes. After the starting chamber is raised, the rat is given $120 \mathrm{~s}$ to explore and the number of explorations per hole is recorded.

Specific procedures used in the present study- On the day before the first trial (experimental day 0), rats underwent a habituation routine to let them get acquainted with the platform and the escape box. In each AT, rats were tested (120 s per trial) with the escape box in place, two times per day for 3 consecutive days (experimental day 1 to 3). On day 4, rats were submitted to a probe trial (PT) during $120 \mathrm{~s}$ without an escape box. In order to eliminate olfactive clues from the maze and the boxes, the surfaces 
were cleaned with $10 \%$ ethylic alcohol solution, after each trial. The behavioral performances were recorded using a computer-linked video camera mounted $110 \mathrm{~cm}$ above the platform. The video-recorded performances of the subjects were measured using the Kinovea v0.7.6 (http://www.kinovea.org) and Image Pro Plus v5.1 (Media Cybernetics Inc., Silver Spring, MD) software. The behavioral parameters assessed were as follows.

(a) Escape box latency: time (in s) spent by an animal since its release from the start chamber until it enters the escape box in the last AT.

(b) Hole exploration frequency during the PT is represented by a bar chart typically bell-shaped around the escape hole. The chart shows 20 bars, each corresponding to a specific hole. For a given hole, the exploratory frequency is the average number of explorations of that hole by either all young (13) or old (11) rats.

(c) Goal sector (GS): Is the area of the platform corresponding to a given number of holes. Thus GS1 is the area corresponding to hole 1; GS3, is the area corresponding to holes $-1,0,+1$. The permanence time in GS3 is calculated taking the time spent by the rats in the area covered by the 3 holes during the PT.

(g) Goal-seeking activity: the sum of explorations for all holes in a PT, divided by 20 (i.e. mean explorations per hole).

\section{Hippocampus Dissection}

Rats were sacrificed by decapitation, the brain was removed carefully, severing the optic nerves and pituitary stalk and placed on a cold plate. The hippocampus was dissected from cortex in both hemispheres using forceps. This dissection procedure was also performed on the anterior and posterior blocks, alternatively placing the brain caudal side up and rostral side up. After dissection, each hippocampus was sagittally halved. One half fixed in PBS buffered 4\% formalin and the second one immediately placed in a $1.5 \mathrm{ml}$ tube and momentarily immersed in liquid nitrogen, then stored at -80 ${ }^{\circ} \mathrm{C}$ for DNA extraction.

\section{Hippocampal DNA extraction}

Hippocampal DNA was extracted using an automated nucleic acid extraction platform called QIAcube HT (Qiagen) with a column-based extraction kit, QIAamp 96 DNA QIAcube HT Kit (Qiagen). 


\section{Purification and methylation analysis of genomic DNA}

DNAs from 24 hemi-hippocampi were purified using the DNeasy Blood and Tissue Kit (Qiagen). Only DNA samples with 260/280 ratios greater than 2.0 were processed for methylation analysis. The bisulfite conversion of genomic DNA was performed using the EZ Methylation Kit (Zymo Research, D5002), following the manufacturer's instructions. The converted genomic DNA was analyzed by Illumina Infinium HorvathMammalMethylChip40 at the UNGC Core Facility. The Horvath Mammal Methyl Chip40 assay provides quantitative measurements of DNA methylation for $22528 \mathrm{CpG}$ dinucleotides that map to the Rattus norvegicus UCSC 6.0 genome (and other mammalian sequences) (17).

\section{Statistical analysis of differentially methylated positions}

Quality control (QC), pre-processing, and statistical analysis of methylation profiles were performed with "minfi" R/Bioconductor package. (18) Briefly, QC analysis was performed with getQC function and further pre-processed with the Noob/ssNoob method.

Data exploratory analysis was performed through an unsupervised hierarchical clustering analysis based on Euclidean distance of methylation profiles. In addition, the distribution of the methylation measurements in each sample was evaluated. To test differential methylation levels in each $\mathrm{CpG}$, multiple hypothesis testing was performed through a linear model using the "limma" package (19). The positive false discovery rate was controlled with a q-value threshold of 0.05 (20).

Gene ontology (GO) enrichment test for positively differentially methylated CpGs from the HorvathMammal40 array was conducted with GOmeth function of "missMethyl" package (21). Gene Ontology gene sets were evaluated, and the significant categories at FDR $<0.05$ reported.

In order to establish potential linear relationship between the methylation of the CpGs present in the statistically significant GO terms and the GS3 permanence time parameter of the Barnes Maze test, we performed linear regression.

\section{Determination of epigenetic age}

In the present study, we used the rat pan-tissue epigenetic clock, which was one of six rat clocks previously described (22). Briefly, the rat pan-tissue epigenetic clock was developed by regressing chronological age on $\mathrm{CpGs}$ from multiple rat tissues that are 
known to map to the genome of Rattus norvegicus. Age was not transformed. Penalized regression models were created with the R function "glmnet" (23). We investigated models produced by both, Ridge and Lasso "elastic net" type regression (alpha=0.5). The optimal penalty parameters in all cases were determined automatically by using a 10 fold internal cross-validation (cv.glmnet) on the training set. By definition, the alpha value for the elastic net regression was set to 0.5 (midpoint between Ridge and Lasso type regression) and was not optimized for model performance. We performed a crossvalidation scheme for arriving at unbiased (or at least less biased) estimates of the accuracy of the DNAm based pan-tissue age estimators. One type consisted of leaving out a single sample (LOOCV) from the regression, predicting an age for that sample, and iterating over all samples.

The final version of the pan-tissue epigenetic clock developed in the above-referenced study was intended for future studies of rat tissue samples and is the clock used here.

\section{RESULTS}

\section{Age-Related DNA methylation differences in the rat hippocampus}

The aim of this analysis was to detect age-related changes in $\mathrm{CpG}$ methylated positions of hippocampal DNA that could be involved in the genesis of neurodegenerative disorders associated with aging. The distribution of the methylation measurements in each sample was evaluated (Suppl. Fig. 1). Data exploratory analysis was performed through an unsupervised hierarchical clustering, where both age groups are distinguishable (Suppl. Fig. 2).

Assessment of each age group showed that the methylation levels of 7222 (32\%) CpGs changed with age between young and old hippocampal samples (q-value<0.05). (Fig. 1). Of these, 1090 (15\%) CpGs exhibited increased methylation (i.e., higher beta values), with age while 5252 (73\%) CpGs showed decreased methylation levels. We identified the $\mathrm{CpGs}$ in context with genomic feature: Promoter, Exon, Intron, Intergenic, 5'UTR, or 3' UTR. The distribution profile of the CpG features in the genome is shown in Fig. 2. The profile of the feature distribution in negatively and positively methylated CpGs is similar and both are similar to the distribution of the feature positions of the Rattus norvegicus CpGs present in the Illumina Infinium HorvathMammalMethylChip40. (Fig. 2, inset). The number of CpGs localized in $\mathrm{CpG}$ islands was $869(12 \%)$. 
In order to identify potential relationships between age-related differences in DNA methylation in the nervous system (hippocampus), we performed an enrichment pathway analysis. Twenty-nine gene sets were significantly enriched in the positively methylated CpGs $(\mathrm{FDR}<0.05)$. Among them, three biological processes are related to the nervous system: neuron fate commitment, brain development, and central nervous system development (Fig. 3) On the contrary, when evaluating negatively methylated CpGs enrichment, no gene set was significantly enriched.

Our last aim in the methylation landscape analysis was to identify the potential correlation between age-related changes in $\mathrm{CpG}$ methylation levels and hippocampusmediated memory performance. In order to determine the relationship between agerelated differences in memory performance and $\mathrm{CpG}$ mehylation levels near genes implicated in nervous system development, we carried out a regression analysis for such CpG sites versus GS3 permanence time index of the Barnes maze test. The reason GS3 permanence was chosen over other Barnes maze parameters is that it reliably reflects spatial memory performance as a whole (also see Discussion).

In the old rat hippocampi, we found that the methylation levels of $14 \mathrm{CpGs}$ correlate negatively with the permanence time in GS3 sector (Fig. 4). Those CpGs are proximal to transcription factors associated with genes Pax5, Lbx1, Nr2f2, Hnf1b, Zic1, Zic4, Hoxd9; Hoxd10, Gli3, Gsx1 and Lmx1b, and Nipbl.

\section{Spatial memory performance of young and old rats in the Barnes maze test}

As expected, all spatial memory indices assessed with the Barnes maze test showed a marked decline with age. Specifically, permanence time in GS1 and GS3 dropped by 88 and $74 \%$ respectively as compared with young counterparts (Fig . 5, panel B). Targetseeking activity, an index of the exploratory initiative of animals, fell by $61 \%$ as compared with young counterparts (Fig. 5 panel C). During the probe trial, the hole exploratory frequency of old rats showed a flatter bell-shaped distribution around hole 0 than young counterparts. The highest difference was detected at hole 0 (93\%) (Fig. 5, panel $D$ and inset, respectively). Latency to escape box, a measure of learning performance and memory retention which is inversely proportional to memory and learning performance, showed a $77 \%$ increase in the old rats versus young counterparts (Fig . 5, panel E). 


\section{Chronological age versus DNAm age}

While chronological age measures the physical time elapsed since rats were born, epigenetic age reflects biological time, that is the biological dynamics of an organism. A regression analysis of hippocampal DNAm age versus chronological age, showed a very high $\mathrm{R}$ value $(\mathrm{R}=0.99)$ using testing data (Fig. 6. panel A). The slope was $<1$ and regression line intersected the $\mathrm{Y}$ axis at nearly 3 months. The same data displayed in a bar chart revealed that in young rats epigenetic time moves faster than physical time (the animals were epigenetically older than indicated by chronological age) but at old ages epigenetic time moves slower that physical time so that while the chronological age of the old group was 26.6 months, their epigenetic ages was only 20.1 months (Fig • 8 panel B). Regression of hippocampal versus blood DNAm age in combined training data from young and old rats revealed a highly significant regression (Suppl. Fig. 3).

\section{Correlation of Barnes maze test indices with hippocampal DNAm age}

A regression analysis between Barnes maze test data and hippocampal DNAm age was performed. In all cases, testing data were used for DNAm age. When data were taken from young and old rats together, a significant regression was found for Exploration frequency of hole $0(\mathrm{R}=0.77)$. For GS1 and GS3 permanence time, the regression was significant in both cases with $\mathrm{R}=0.72$ and 0.79 , respectively (Fig. 7 panels $\mathbf{A}, \mathbf{B}$ and $\mathbf{C}$ ). When the regressions were done using data from either young or old rats alone, no significant regression was found (data not shown).

Target-Seeking activity showed a significant regression for hippocampal DNAm age with $\mathrm{R}=0.70$ (Fig. 10, panel B). Latency time also showed a significant regression with hippocampal DNAm age with $\mathrm{R}=0.72$. (Fig. 10, panel A). As before, when the regressions were done using data from either young or old rats, no significance was detected (data not shown).

\section{DISCUSSION}

DNA methylation is one of the best understood modifications of chromatin, with crucial roles in gene expression and imprinting, defense against viral sequences, inhibition of recombination, as well as assembly of heterochromatin (24). Three active DNA methyltransferase (DNMT) activities (DNMT1, DNMT3a, and DNMT3b) have been identified in humans and mice (25-26). DNMT1 functions primarily as maintenance DNMT, responsible for copying the parental-strand DNA methylation pattern onto the 
daughter strand after each round of DNA replication. DNMT3a and DNMT3b function as de novo DNMTs, although they can also maintain methylation patterns. DNA methylation is associated with the induction of a closed chromatin state by recruitment of protein complexes that bear repressive chromatin modifying activities, including MBD (methyl-CpG-binding domain) proteins and histone deacetylase (HDAC)containing complexes (27-28). Cytosine methylation at euchromatic promoters participates in switching off the corresponding genes. At heterochromatic domains, it participates in the assembly of the highly compacted chromatin characteristic of these domains.

\section{DNA demethylation in the old rat hippocampus}

In the brain, DNA methylation regulates neural activities and memory formation via the control of gene expression in neurons (29). Early studies showed that a global loss of DNA methylation occurs in the rat brain during aging (24). In line with these finding, our results reveal that in the rat hippocampus aging is associated with predominant DNA demethylation ( $73 \%$ versus $15 \%$ hypermethylation). Results in rat brain are consistent with the more general observation that, almost invariably, mammalian aging is more commonly associated with $\mathrm{CpG}$ hypomethylation, particularly at repetitive DNA sequences (30-33). This is likely to be at least partly responsible for the loss of heterochromatin during aging. In the hippocampus, aging does not appear to have a significant impact on the methylation levels of $\mathrm{CpG}$ islands as only $12 \%$ of the differentially methylated CpGs was localized in these DNA regions.

\section{Increase of hippocampal DNAm in genes of the Zic and Gli families in old rats}

In the old rats the results of our GO analysis identified a number of genes that are hypermethylated during aging in the rat hippocampus. In functional terms, the more relevant of the set are the hypermethylated genes of the Zic and Gli families, Zic1, Zic4 and Gli3.

The Zinc finger of the cerebellum genes Zic 1 and Zic4, and the Gli Family Zinc Finger 3 Gli3 play specific roles in development (34-35). Zic1, Zic4, and Gli3 gene expression has been found downregulated in the aged hippocampus of Sprague Dawley rats (36). It has been reported that the proteins of the Zic and Gli families interact and regulate each other's transcription through their respective zinc finger domains. Moreover, Zic 1 contributes to the translocation of Gli3 from the cytoplasm to the nucleus (37). 
Gli proteins are part of the Hedgehog Signalling pathway, they act as transcription factor activators (or repressors, in absence of the Sonic Hedgehog protein) on the target genes in the last step of the pathway (38). Several studies carried out in multiple species concluded that Zic absence can repress Sonic hedgehog (Shh)-signaling, probably by the interaction between Zic and Gli proteins (39). Shh signaling was suggested to be involved in age-related neurological disorders since it mediates the formation and plasticity of neuronal circuits (40). Thus, our results suggest that the increase in the $\mathrm{CpG}$ methylation of these genes in the hippocampus of old rats may have a negative impact on the cognitive performance of the animals, possibly through the effects of the Shh signalling pathway on hippocampal-mediated memory.

\section{Identification of genes near or at hippocampal GpGs whose methylation levels correlate inversely with GS3 permanence time in old rats}

As mentioned above, experience has shown that from the different spatial memory indices the Barnes maze test generates, GS3 permanence time is the one that most reliably quantitates spatial memory performance. For instance, after treatment of 27 months old female rats with naïve or insulin-like growth factor-1 (IGF-1) transgenic human umbilical cord cells, the only Barnes maze indices that detected an improvement in spatial memory in the treated animals were permanence in GS1 and GS3 sectors which increased significantly whereas there were no changes in intact counterparts (41).

Our finding that in old rats of the same chronological age (26.6 mo.) there is a set of 14 hypermethylated CpGs near or at specific genes whose methylation levels are significantly and inversely associated with spatial memory function (as indicated by GS3 permanence time) suggests, although does not prove, that the methylation levels of those CpGs are causally involved in the observed age-related impairment of spatial memory function. The hypothesis is strengthened by the fact that a number of those 14 CpGs that correlate significantly with GS3 permanence time are proximal to genes of the Zic and Gli families. Since the Zic and Gli genes are involved in nervous system development, it is conceivable that during the whole lifetime of rats, the methylation levels of those CpGs increase continuously, decreasing the expression of hippocampal genes important for spatial memory performance. Our results are a descriptive observation in untreated rats. 
If an experimental manipulation that slows down aging (i.e., a life extending treatment) were associated with a decrease in methylation levels of those 14 hippocampal CpGs (when compared with untreated controls of the same age), the idea that those CpGs play a causal role in spatial memory performance would be further favored. The observation that the highest $\mathrm{R}^{2}$ value, 0.85 , observed in this analysis corresponds to CpG 07225115 , which is located near the Zic1 gene is in line with the above hypothesis (Fig. 6 panel J).

\section{Hippocampal DNAm age and spatial memory performance in rats}

Considering again the genesis of the epigenetic clock, it is of interest to mention that in 2013 Horvath's algorithm was successfully tested using approximately 8,000 human DNA methylation data sets from over 30 different tissue types (11). For a given chronological age, it was found that in DNA samples taken from whole blood, peripheral blood mononuclear cells, buccal epithelium, colon, adipose, liver, lung, saliva, and uterine cervix, Horvath's algorithm read essentially the same epigenetic age, the only exceptions being some brain regions and very few other organs $(\mathbf{1 1}, \mathbf{4 2}, \mathbf{4 3})$. In healthy humans, the cerebellum is epigenetically younger than the rest of the organism (44). Multi-tissue full lifespan epigenetic clocks have been set up for mice $(\mathbf{1 5}, \mathbf{4 5})$ and more recently, for Fischer344 male rats (46).

In a recent study we generated multiple epigenetic clocks for Sprague Dawley (S-D) male and female rats including the design of a pan tissue epigenetic clock for this species (22). Using this new tool, the rate of epigenetic aging of peripheral tissues including liver, ovary, skin, adipose tissue, and blood showed comparable features to those reported in humans and mice $(\mathbf{1 1}, \mathbf{1 5}, \mathbf{4 5})$. The rate of epigenetic aging in a number of brain regions, including the neocortex, cerebellum, hippocampus, substantia nigra, hypothalamus and anterior pituitary were also assessed in S-D females, with the rat pan-tissue clock. Among these six regions, the hypothalamus and pituitary gland showed a marked departure from the average rate of epigenetic aging of the rest of the tissues. In both pituitary gland and hypothalamus, the rate of epigenetic aging was substantially slower than the average rate for other brain regions and peripheral tissues (22). It is of interest that, using training data, blood DNAm age showed a highly significant regression with hippocampal DNAm age (suppl. Fig. 1). This is in line with a previous study showing a significant correlation between human brain and blood DNAm modules, which led the authors to conclude that blood is a promising surrogate for brain tissue when studying the effects of age on DNA methylation profiles (44). 
Our present results reveal that the functional performance of several spatial memoryrelated indices show significant regression levels when plotted versus hippocampal DNAm age only when data from young and old rats are taken together. In no case regressions of single age groups were significant.

Since the hypermethylation levels of a group of 14 hippocampal CpGs in old rats correlated significantly with spatial memory performance, and epigenetic age is thought to reflect biological rather than chronological age, we expected that regression of different Barnes maze indices versus hippocampal DNAm age in old rats would reveal a significant association. The fact that no regression was significant suggests that hypermethylation of certain hippocampal CpGs may play a more relevant role in memory function than DNAm age.

The observation that hippocampal epigenetic time moves faster than physical time at younger ages but the rate of epigenetic time becomes slower than physical time in old rats is consistent with the fact that developmental changes are faster in young than old animals. The observation is in line with the DNAm age data previously reported in different brain regions of rats of various ages (22).

\section{CONCLUDING REMARKS}

The recent development of the Illumina Infinium HorvathMammalMethylChip40, a mammalian methylation array that possesses a high fidelity in humans, rats, and mice (17), has made it possible to profit from a number of advantages that offers the rat as an animal model. For instance its size which is 10 times larger than mouse size is a feature that allows to extract sufficient tissue DNA from small brain regions as the hippocampus. Thus, in rats, we were able to quantitatively assess epigenetic age and other DNAm-related features during aging and rejuvenation (22). In this context, the present results, focused on hippocampal DNAm, show that hippocampal DNAm age is a reliable index of spatial memory performance in young and old rats. Perhaps more significant are the data that come from the GO enrichment and regression analysis in old rats as they suggest that age-related hypermethylation of certain gene families like Zic and Gli may play a causal role in the decline in spatial memory during aging. This is to our knowledge, the first documented study in experimental animals linking age-related changes in hippocampal methylation levels of particular genes with the decline in spatial memory during aging. Our results offer valuable reference values for future 
epigenetic studies assessing the effectiveness of life-extending or rejuvenation strategies in this species.

\section{ACKNOWLEDGEMENTS}

The authors thank Dr. Kenneth Raj, Public Health England, Didcot, UK, for enlightening discussions on the epigenetic clock and Dr. Martin Abba, CINIBA, UNLP, Argentina for technical comments on methylation analysis. The authors are indebted to Mr. Mario R. Ramos for design of the figures, to Ms. Yolanda E. Sosa for technical and editorial assistance and to Ms. Araceli Bigres for excellent care of our rat colony.

This study was supported in part by grant PICT18 - 00907, from the National Agency for the Promotion of Science and Technology, Argentina and from research grant MRCF 10-10-17 from the Medical Research Charitable Foundation and the Society for Experimental Gerontological Research, New Zealand to RGG, grant PICT 2018-2446 to $\mathrm{CBH}$ and grant \#PICT16-1070 to GRM. SH was supported by the Paul G. Allen Frontiers Group.

Author Approvals: All authors have seen and approved the manuscript, and that it hasn't been accepted or published elsewhere.

Competing Interests: None of the authors has potential competing interests to disclose. 


\section{REFERENCES}

1- $\quad$ Squire LR, Knowlton B, Musen G. The structure and organization of memory. Annu Rev Psychol 44: 453-495 (1993)

2- $\quad$ O'Keefe J, Nadel L. The Hippocampus as a Cognitive Map. Oxford: Oxford University Press (1976).

3- Morel GR, Andersen T, Pardo J, Zuccolilli GO, Cambiaggi VL, Hereñú CB, Goya RG. Cognitive impairment and morphological changes in the dorsal hippocampus of very old female rats. Neuroscience 303: 189-199 (2015).

4- Blalock EM, Chen KC, Sharrow K, Herman JP, Porter NM, Foster TC, Landfield PW. Gene microarrays in hippocampal aging: statistical profiling identifies novel processes correlated with cognitive impairment. J Neurosci 23 : 3807-3819 (2003).

5- $\quad$ Burger C, López MC, Feller JA, Baker HV, Muzyczka N, Mandel RJ. Changes in transcription within the CA1 field of the hippocampus are associated with age-related spatial learning impairments. Neurobiol Learn Mem 87 : 21-41 (2007).

6- $\quad$ Burger C, Lopez MC, Baker HV, Mandel RJ, Muzyczka N. Genome-wide analysis of aging and learning-related genes in the hippocampal dentate gyrus. Neurobiol Learn Mem 89 (4): 379-96 (2008).

7- $\quad$ Rowe WB, Blalock EM, Chen KC, Kadish I, Wang D, Barrett JE, Thibault O, Porter NM, Rose GM, Landfield PW. Hippocampal expression analyses reveal selective association of immediate-early, neuroenergetic, and myelinogenic pathways with cognitive impairment in aged rats. J Neurosci $27: 3098-3110$ (2007)

8- Verbitsky M, Yonan AL, Malleret G, Kandel ER, Gilliam TC, Pavlidis P. Altered hippocampal transcript profile accompanies an age-related spatial memory deficit in mice. Learn Mem $11: 253-260$ (2004)

9- $\quad$ Pardo J, Abba M, Lacunza E, Francelle L, Morel GR, Outeiro TF, Goya RG. Identification of a conserved gene signature associated with an exacerbated inflammatory environment in the hippocampus of aging rats. Hippocampus 27: 435-449 (2017).

10- Vanyushin BF, Nemirovsky LE, Klimenko VV, Vasiliev VK, Belozersky AN. The 5-methylcytosine in DNA of rats. Tissue and age specificity and the changes induced by hydrocortisone and other agents. Gerontologia 19: 138-152, (1973).

11- Horvath S: DNA methylation age of human tissues and cell types; Genome Biol 14: R115-R133 (2013).

12- Bocklandt, S, Lin W, Sehl ME, Sánchez FJ, Sinsheimer JS, Horvath S, Vilain E. Epigenetic predictor of age. PLoS One 6(6): e14821 (2011).

13- Hannum, G., Guinney J, Zhao L, Zhang L, Hughes G, Sadda S, Klotzle B, Bibikova M, Fan JB, Gao Y, Deconde R, Chen M, Rajapakse I, Friend S, Ideker $\mathrm{T}$, Zhang K. Genome-wide methylation profiles reveal quantitative views of human aging rates. Mol Cell 49: 359-367 (2013).

14- Weidner, C.I; Lin Q, Koch CM, Eisele L, Beier F, Ziegler P, Bauerschlag DO, Jöckel KH, Erbel R, Mühleisen TW, Zenke M, Brümmendorf TH, Wagner W. Aging of blood can be tracked by DNA methylation changes at just three $\mathrm{CpG}$ sites. Genome Biol 15(2): R24 (2014).

15- Meer MV, Podolskiy DI, Tyshkovskiy A, Gladyshev VN; A Whole Lifespan Mouse Multi-Tissue DNA Methylation Clock; Elife; 14;7:e40675. doi: 10.7554/eLife.40675 (2018). 
16- Choi, H., S. Joe, and H. Nam, Development of Tissue-Specific Age Predictors Using DNA Methylation Data. Genes (Basel); 10(11) (2019).

17- Arneson A,Haghani A, Thompson MJ, Pellegrini M, Kwon SB, Vu H, Li CZ, Lu AT, Barnes B, Hansen KD, Zhou W, Breeze CE, Ernst J, Horvath S. A mammalian methylation array for profiling methylation levels at conserved sequences. bioRxiv 2021.01.07.425637; doi:

https://doi.org/10.1101/2021.01.07.425637

18- Aryee MJ, Jaffe AE, Corrada-Bravo H, Ladd-Acosta C, Feinberg AP, Hansen KD, Irizarry RA (2014). "Minfi: A flexible and comprehensive Bioconductor package for the analysis of Infinium DNA Methylation microarrays." Bioinformatics, 30(10), 1363-1369. doi: 10.1093/bioinformatics/btu049.

19 - Ritchie ME, Phipson B, Wu D, Hu Y, Law CW, Shi W, Smyth GK (2015). "limma powers differential expression analyses for RNA-sequencing and microarray studies." Nucleic Acids Research, 43(7), e47. doi: 10.1093/nar/gkv007.

20 - Benjamini, Y. and Hochberg, Y. (1995), Controlling the False Discovery Rate: A Practical and Powerful Approach to Multiple Testing. Journal of the Royal Statistical Society: Series B (Methodological), 57: 289-300. https://doi.org/10.1111/j.2517-6161.1995.tb02031.x. (1995), Controlling the False Discovery Rate: A Practical and Powerful Approach to Multiple Testing. Journal of the Royal Statistical Society: Series B (Methodological), 57: 289-300. https://doi.org/10.1111/j.2517-6161.1995.tb02031.x

21- Phipson B, Maksimovic J, Oshlack A (2015). "missMethyl: an R package for analysing methylation data from Illuminas HumanMethylation450 platform." Bioinformatics, btv560.

22- Horvath S, Singh H , Raj K, Khairnar S, Sanghavi A, Shrivastava A, Zoller JA, Li CZ, Herenu CB, Canatelli-Mallat M, Lehmann M, Solberg Woods LC, Garcia Martinez A, Wang T , Chiavellini P , Levine AJ, Chen H , Goya RG , Katcher HL. Reversing age: dual species measurement of epigenetic age with a single clock; (2020) BioRxiv doi: https://doi.org/10.1101/2020.05.07.082917

23- Friedman, J., Hastie, T., and Tibshirani, R. (2010). Regularization Paths for Generalized Linear Models via Coordinate Descent. Journal of Statistical Software 33, 1-22.

24- Bird A. DNA methylation patterns and epigenetic memory. Genes Dev 16: 6-21 (2002).

25- $\quad$ Okano M, Bell DW, Haber DA, Li E. DNA methyltransferases Dnmt3a and Dnmt3b are essential for de novo methylation and mammalian development. Cell 99: 247-257 (1999).

26- Okano M, Xie S, Li E. Cloning and characterization of a family of novel mammalian DNA (cytosine-5) methyltransferases. Nat Genet 19: 219-220 (1998) 
27- Ballestar E, Esteller M. Methyl-CpG-binding proteins in cancer: blaming the DNA methylation messenger. Biochem Cell Biol 83: 374-384 (2005).

28- Klose RJ, Bird AP. Genomic DNA methylation: the mark and its mediators. Trends Biochem Sci 31: 89-97 (2006).

29- Liu L, van Groen T, Kadish I and Tollefsbol, TO; DNA methylation impacts on learning and memory in aging; Neurobiol Aging 30(4): 549-560 (2009).

30- Bjornsson HT, Sigurdsson MI, Fallin MD, Irizarry RA, Aspelund T, Cui H, Yu W, Rongione MA, Ekström TJ, Harris TB, Launer LJ, Eiriksdottir G, Leppert MF, Sapienza C, Gudnason V, Feinberg AP. Intra-individual change over time in DNA methylation with familial clustering. JAMA 299:2877-2883 (2008).

31- Bollati V, Schwartz J, Wright R, Litonjua A, Tarantini L, Suh H, Sparrow D, Vokonas P, Baccarelli A. Decline in genomic DNA methylation through aging in a cohort of elderly subjects. Mech Age Dev 130:234-239 (2009).

32- Bormann F, Rodríguez-Paredes M, Hagemann S, Manchanda H, Kristof B, Gutekunst J, Raddatz G, Haas R, Terstegen L, Wenck H, Kaderali L, Winnefeld M, Lyko F. Reduced DNA methylation patterning and transcriptional connectivity define human skin aging. Aging Cell 15:563-571 (2016).

33- Christensen BC, Houseman EA, Marsit CJ, Zheng S, Wrensch MR, Wiemels JL, Nelson HH, Karagas MR, Padbury JF, Bueno R, Sugarbacker DJ, Yeh RF, Wiencke JK, Kelsey KT. Aging and environmental exposures alter tissuespecificDNA methylation dependent upon $\mathrm{CpG}$ island context. PLOS Genet 5:e1000602 (2009).

34- Aruga, J. (2004). The role of Zic genes in neural development. Molecular and Cellular Neuroscience, 26(2), 205-221.

35- Hui, C. C., \& Angers, S. (2011). Gli proteins in development and disease. Annual review of cell and developmental biology, 27, 513-537.

36- Shavlakadze T, Morris M, Fang J, Wang SX, Zhu J, Zhou W, Tse HW, Mondragon-Gonzalez R, Roma G, Glass DJ. Age-Related Gene Expression Signature in Rats Demonstrate Early, Late, and Linear Transcriptional Changes from Multiple Tissues. Cell Rep. 2019 Sep 17;28(12):3263-3273.e3. doi: 10.1016/j.celrep.2019.08.043. PMID: 31533046.

37- Koyabu Y, Nakata K, Mizugishi K, Aruga J, Mikoshiba K. Physical and functional interactions between Zic and Gli proteins. J Biol Chem. 2001 Mar 9;276(10):6889-92. doi: 10.1074/jbc.C000773200. Epub 2001 Jan 12. PMID: 11238441 .

38- Jacob, J., \& Briscoe, J. (2003). Gli proteins and the control of spinal cord patterning. EMBO reports, 4(8), 761-765.

39- Bellchambers HM, Ware SM. ZIC3 in Heterotaxy. Adv Exp Med Biol. 2018;1046:301-327. doi: 10.1007/978-981-10-7311-3_15. PMID: 29442328.

40- Yao, P. J., Petralia, R. S., \& Mattson, M. P. (2016). Sonic Hedgehog Signaling and Hippocampal Neuroplasticity. Trends in neurosciences, 39(12), 840-850. https://doi.org/10.1016/j.tins.2016.10.001

41- Lehmann M, Zappa-Villar MF, García MG, Mazzolini G, Canatelli-Mallat M, Morel GR, Reggiani PC, Goya RG.; Umbilical Cord Cell Therapy Improves Spatial Memory in Aging Rats; Stem Cell Reviews and Reports; doi: 10.1007/s12015-019-09895-2. (2019).

42- Horvath, S., Raj, K. DNA methylation-based biomarkers and the epigenetic clock theory of ageing. Nat. Rev. Genet. 19, 371-384 (2018). 
43- Raj, K., 2018. The Epigenetic Clock and Ageing, in: Moskalev, A., Vaiserman, A.M. (Eds).Epigenetics of Aging and Longevity, From the series Translational Epigenetics vol 4. Academic Press, New York, pp 95-118.

44- Horvath S, Zhang Y, Langfelder P, Kahn RS, Boks MP, van Eijk K, van den Berg LH, Ophoff RA. Aging effects on DNA methylation modules in human brain and blood tissue. Genome Biol. 2012 Oct 3;13(10):R97. doi: 10.1186/gb2012-13-10-r97.

45- Thompson MJ, Chwiałkowska K, Rubbi L, et al. A multi-tissue full lifespan epigenetic clock for mice. Aging (Albany NY). 2018; 10:2832-2854. doi:10.18632/aging.101590.

46- Levine M, McDevitt RA, Meer M, Perdue K, Di Francesco A, Meade T , Farrell C, Thrush K, Wang M, Dunn C, Pellegrini M, de Cabo R, Ferrucci L. A rat epigenetic clock recapitulates phenotypic aging and co-localizes with heterochromatin; Elife 9:e59201. doi: 10.7554/eLife.59201 (2020) 


\section{MAIN FIGURES}
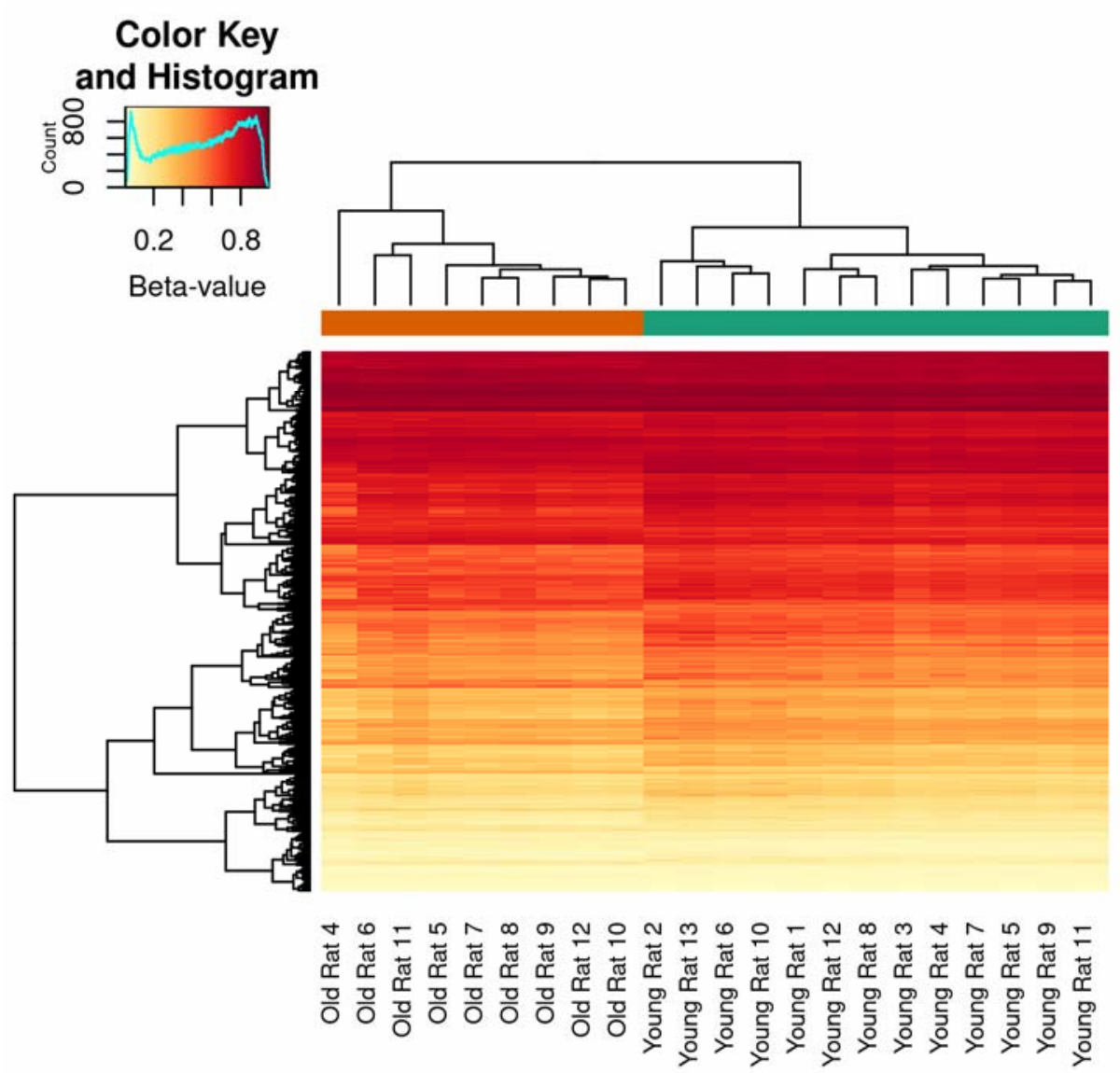

Figure 1- Heatmap of differentially methylated CpG sites in the old group compared with the young one. Yellow denotes CpGs with the lowest methylation levels (beta values close to 0 ) and red denotes CpGs with the highest methylation levels (beta values close to 1).

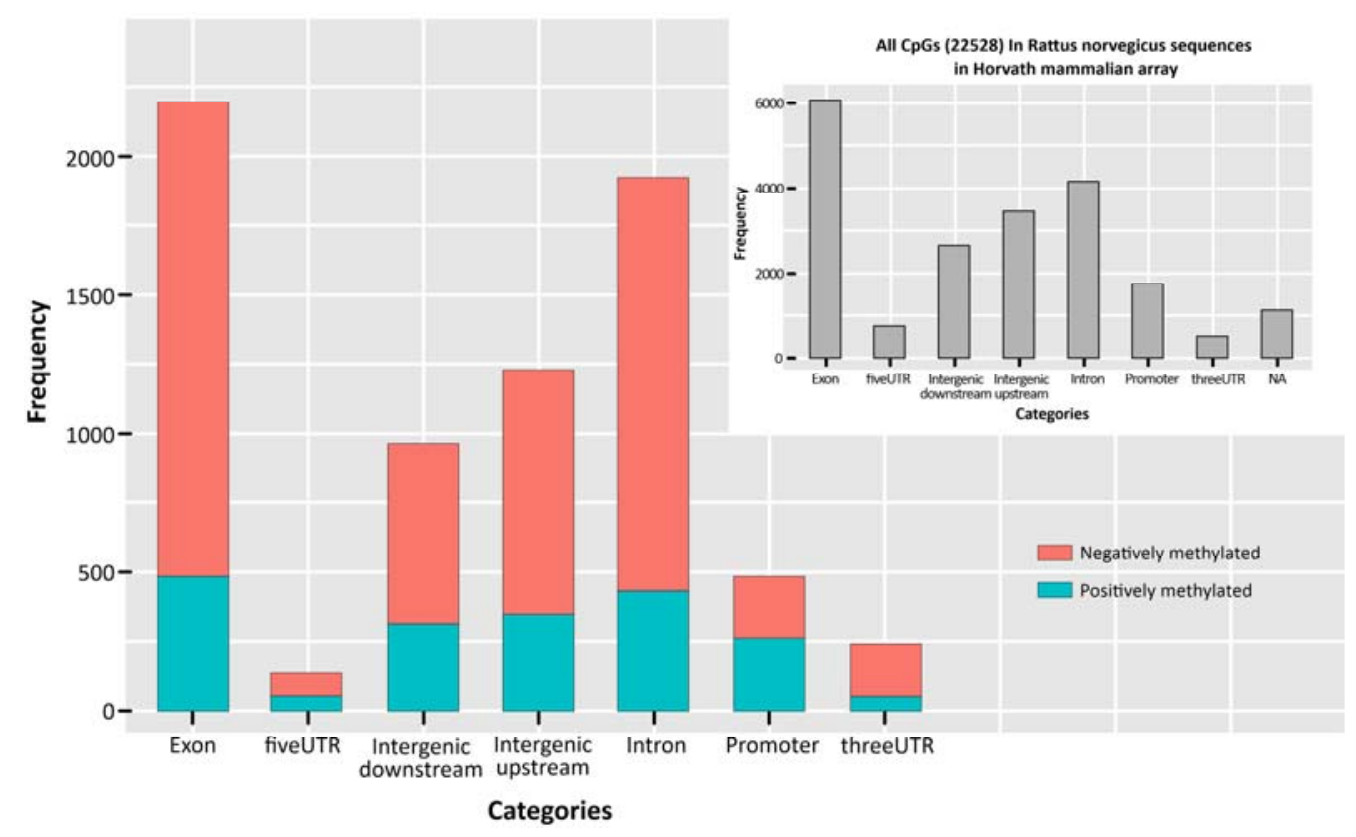


bioRxiv preprint doi: https://doi.org/10.1101/2021.05.07.443204; this version posted May 9, 2021. The copyright holder for this preprint (which was not certified by peer review) is the author/funder, who has granted bioRxiv a license to display the preprint in perpetuity. It is made available under aCC-BY-ND 4.0 International license.

Figure 2- Genomic feature positions of the differentially methylated CpGs. Merged barchart of the genomic locations for the age-dependent positively methylated CpGs (in color1) and the negatively methylated CpGs (in color2). Inset- Feature distribution of the Rattus norvegicus sequences in the Illumina Infinium Horvath Mammal Methyl Chip40. neuron fate commitment
skeletal system developmen
brain developmen
embryonic morphogenesis transcription regulatory region sequence-specific DNA binding cis-regulatory region sequence-specific DNA binding RNA polymerase II cis-regulatory region sequence-specific DNA binding regulatory region nucleic acid binding embryonic skeletal system development embryonic skeletal system morphogenesis central nervous system development regionalization transcription by RNA polymerase II DNA-binding transcription activator activity, RNA polymerase II-specificDNA-binding transcription activator activityregulation of transcription by RNA polymerase II cell fate commitment RNA polymerase II transcription regulatory region sequence-specific DNA binding transcription regulator activity molecular function regulator sequence-specific DNA binding double-stranded DNA binding. pattern specification process animal organ morphogenesis sequence-specific double-stranded DNA binding DNA-binding transcription factor activity. DNA-binding transcription factor activity, RNA polymerase II-specific

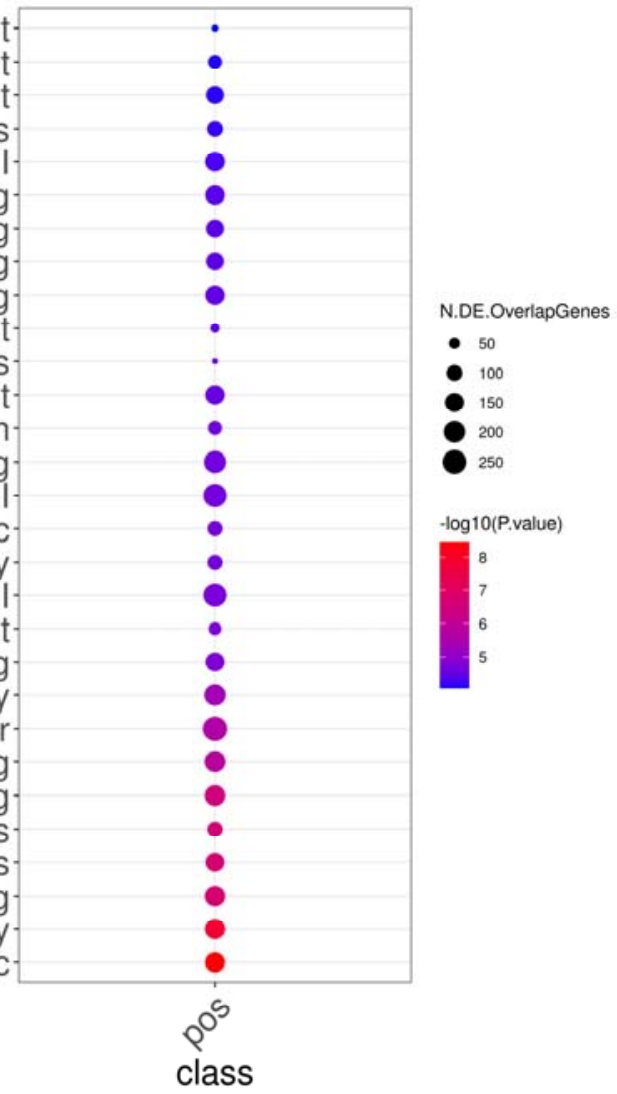

Figure 3- Gene ontology enrichment for positively methylated CpGs. Dot plots for significant gene sets with FDR $<0.05$ are displayed. Circle size represents the number of genes related to significant CpGs in a particular set. 

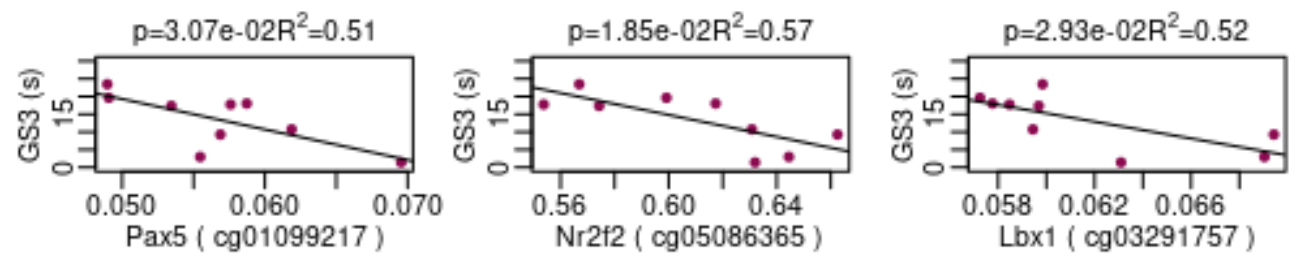

$p=1.13 e-02 R^{2}=0.62$
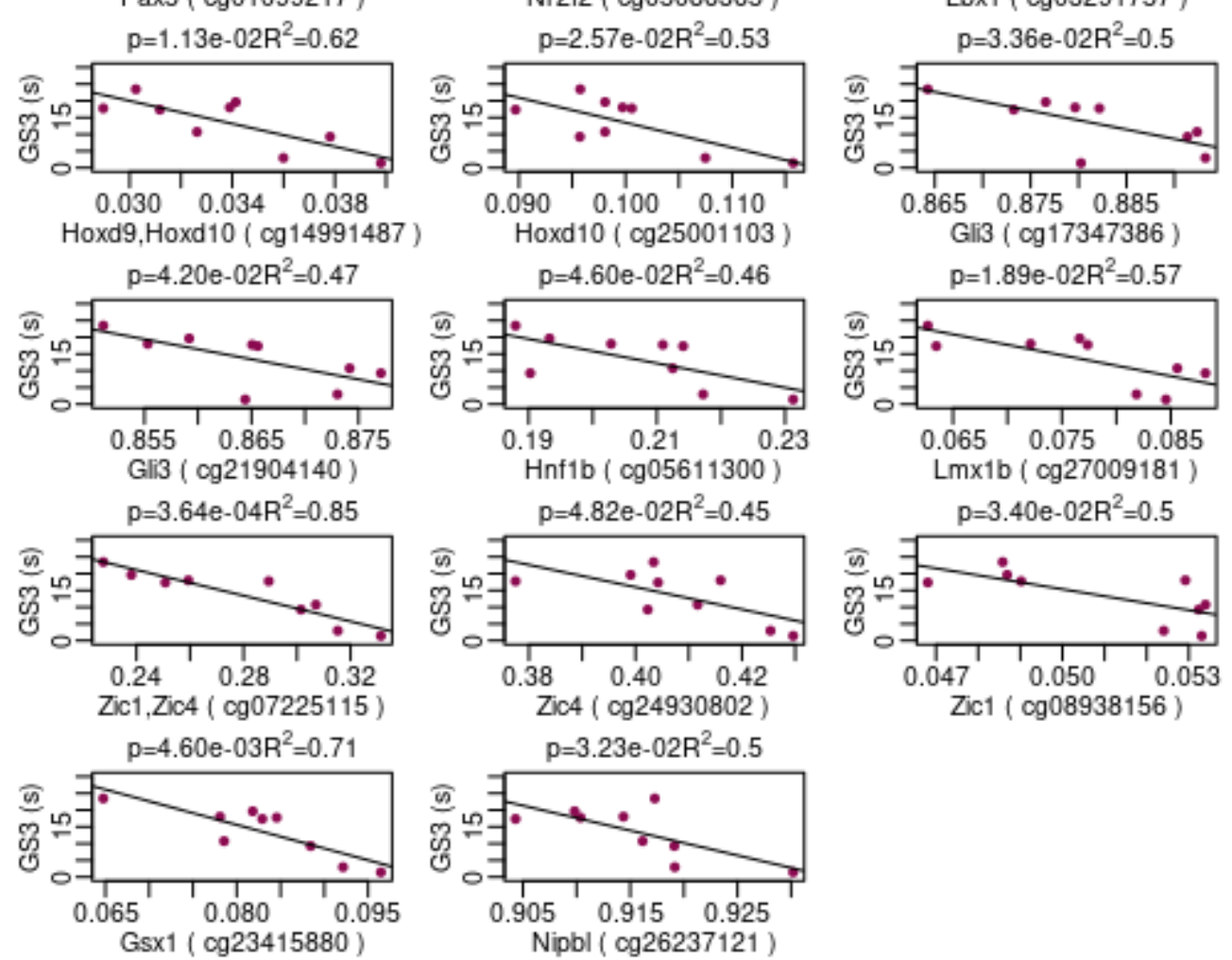

Zic1 ( cg08938156)

Figure 4- Regression plots correlating Beta-value from CpGs in the central nervous system enrichment versus permanence time in GS3 Barnes maze sector- In old rat hippocampi, methylation levels of $14 \mathrm{CpGs}$ correlate negatively with permanence time in the GS3 sector. The CpGs are proximal to transcription factors associated with genes Pax5, Lbx1, Nr2f2, Hnf1b, Zic1, Zic4, Hoxd9; Hoxd10, Gli3, Gsx1 and Lmx1b, and Nipbl. 
Spatial memory in young and old rats.

Barnes maze test parameters
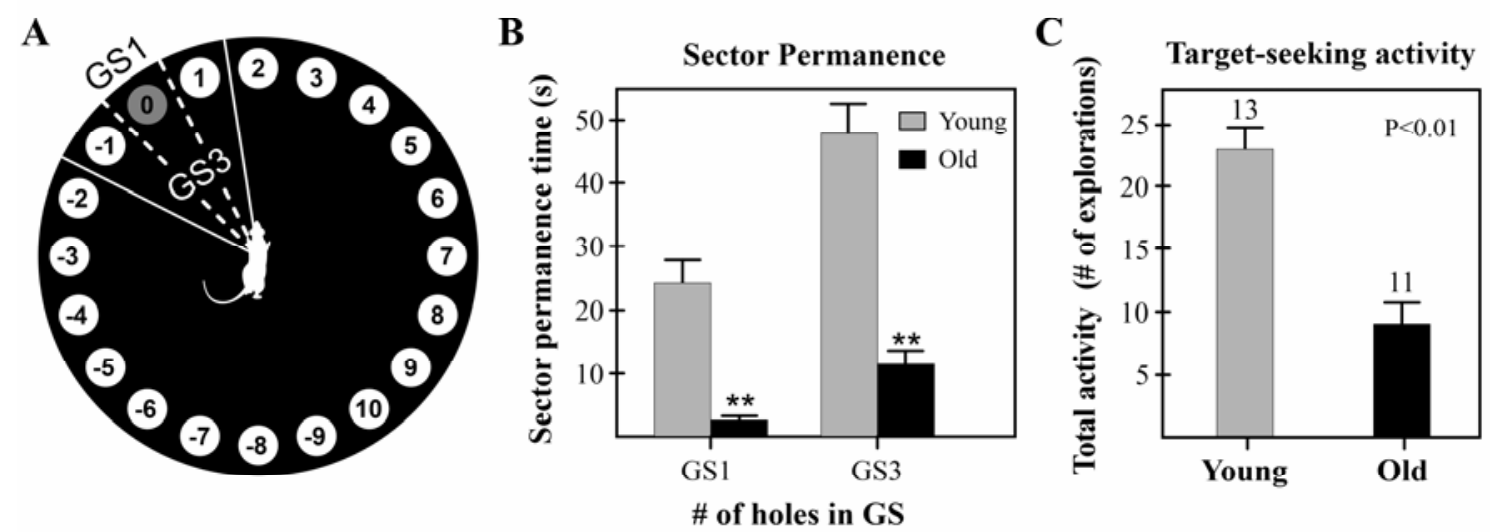

D Hole Exploration Frequency

$\mathbf{E}$

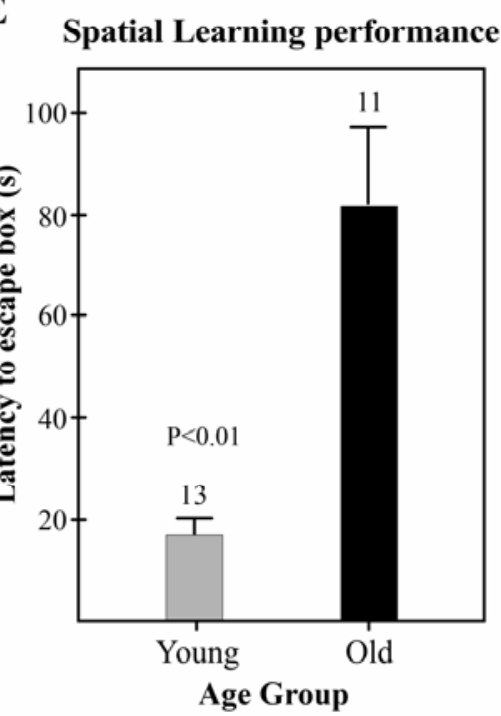

Figure 5- Summary of spatial memory parameters in young and old female ratsPanel A- Barnes maze platform showing the outlines of goal sector 1 (GS1) and goal sector 3 (GS3). Panel B- Impact of aging on GS1 and GS3 permanence. Sector permanence is expressed as the time spent in the corresponding sector during the testing time (2 min). Panel C- Target (escape box)-seeking activity in young and old animals. Panel D- Hole exploration frequency in young and old rats. Inset- Number of explorations of hole 0 (escape hole) in young and old rats during the testing time.

Panel E- Effect of aging on learning and spatial memory retention in rats. Columns represent escape hole latency (seconds it takes for rats to find the escape hole when the box is in place) at the end of the training. 


\section{A \\ Chronological vs DNAm age}

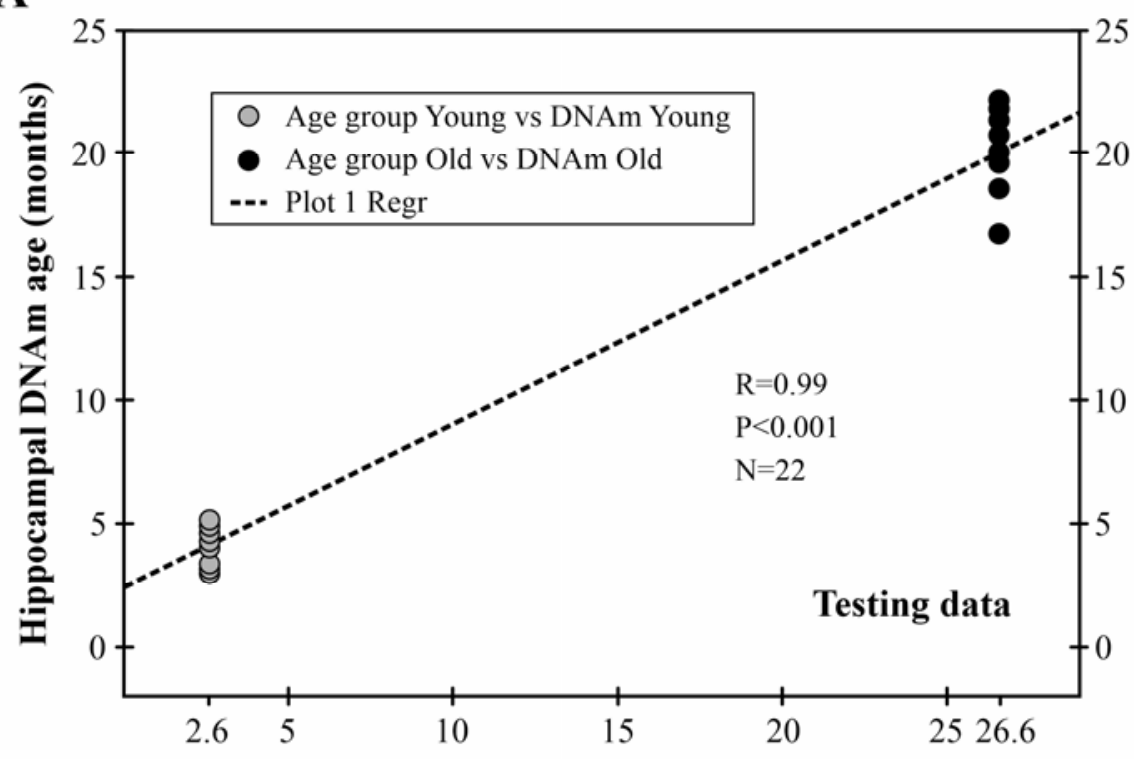

Chronological age (months)

B

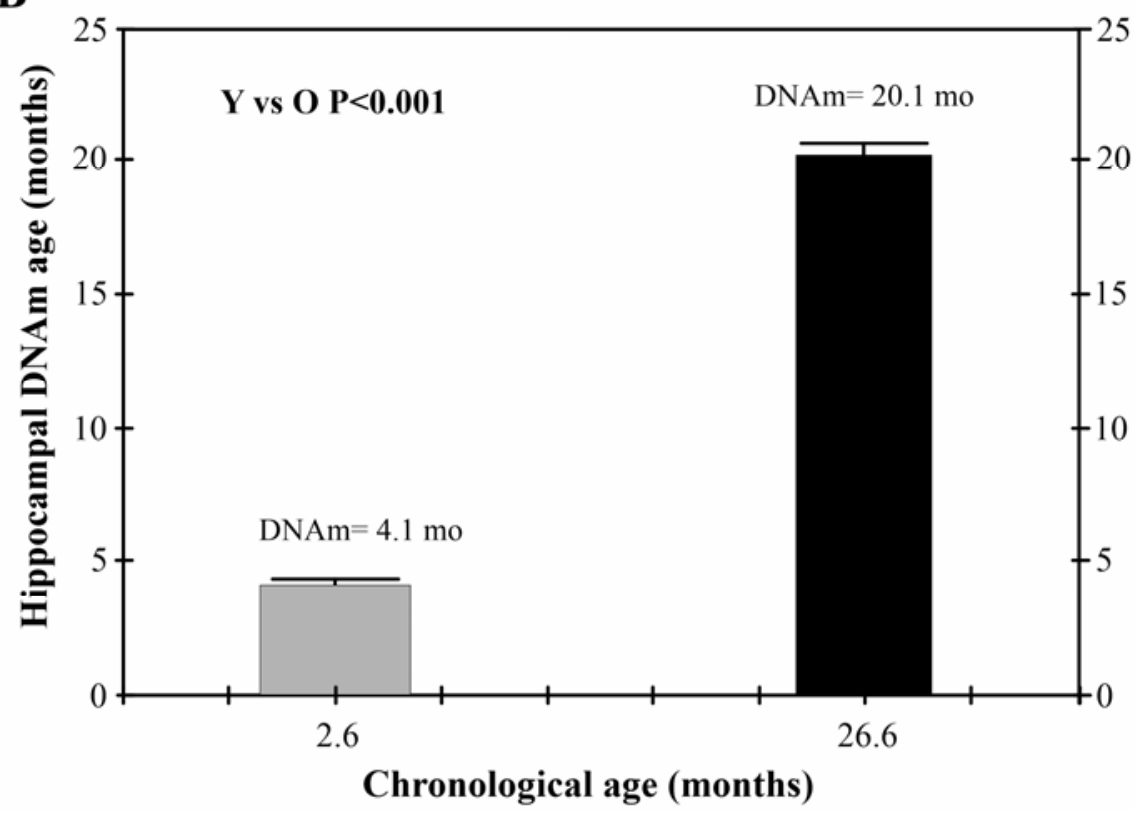

Figure 6- Correlation of hippocampal DNAm age verus chronological age in female rats- Panel A- Regression plot of hippocampal DNA, versus chronological age. Notice the although there is a high correlation between the two types of age, the slope is not $=1$, that is, they are not identical Panel B- The same plot is drawn as a bar chart. Notice that at young ages the epigenetic clock ticks faster than the physical clock. In old animals, the ticking of the DNAm clock is slower than that of physical time. 


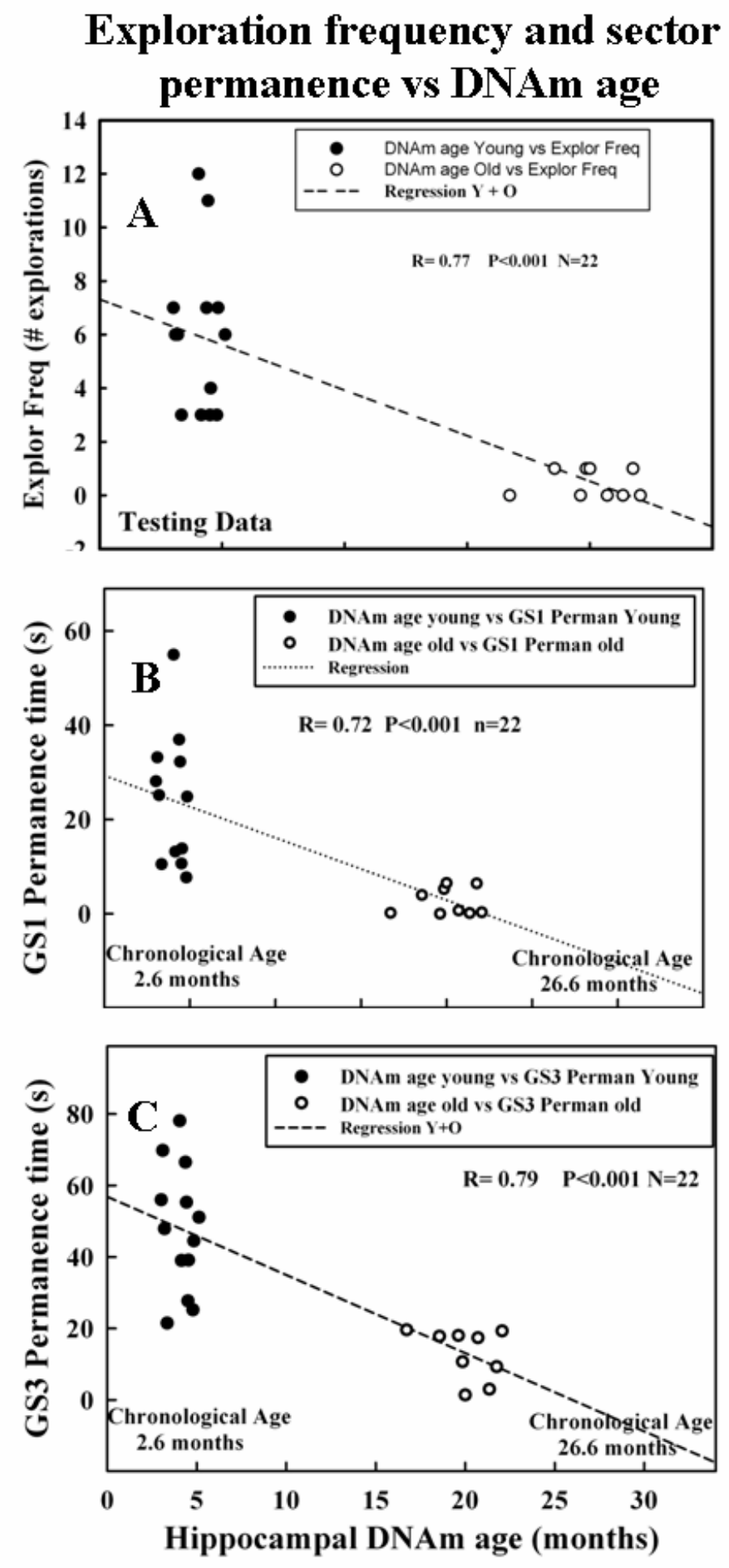

Figure 7- Regression plots correlating spatial memory variables measured by the Barnes maze test versus hippocampal DNAm age. Panel A- Regression of hole exploration frequency versus hippocampal DNAm age in young and old rats. The regression is highly significant. Panel B- Time spent by rats in goal sector 1 plotted versus hippocampal DNAm age. In this case, the regression is also highly significant. Panel C- Same plot for GS3 permanence. The regression also is highly significant here. 

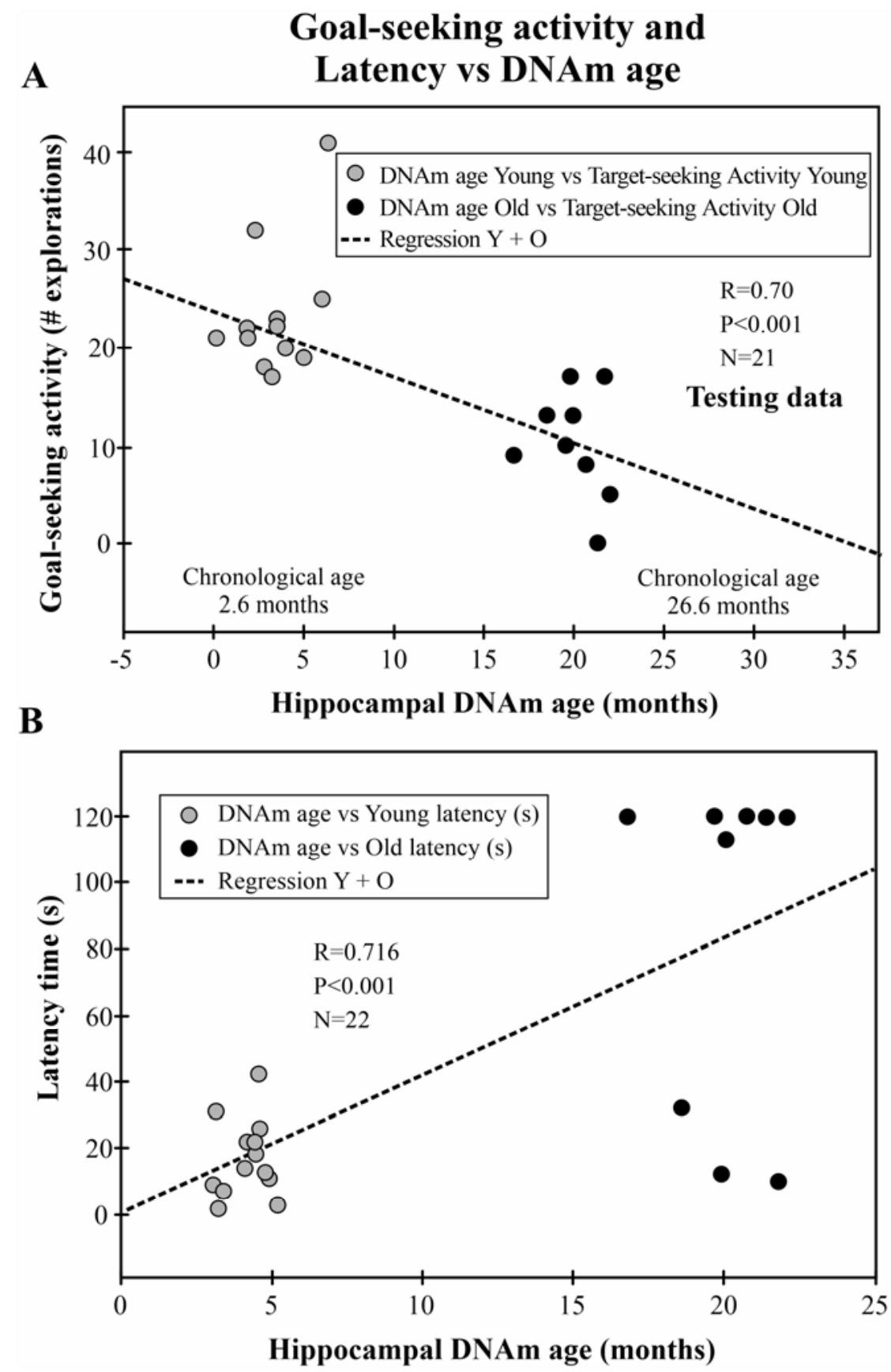

Figure 8- Regression plots correlating cognitive indices versus hippocampal DNAm age. Panel A- All-hole exploratory activity versus hippocampal DNAm age. The regression coefficient is highly significant. Panel B- Latency time versus hippocampal DNAm age. Latency is an index of learning ability and spatial memory retention. The regression of the plot is highly significant. In all panels, asterisks over bars indicate a highly significant difference from corresponding young counterparts. Numbers over bars indicate the $\mathrm{N}$ value for the corresponding group. 


\section{SUPPLEMENTAL FIGURES}

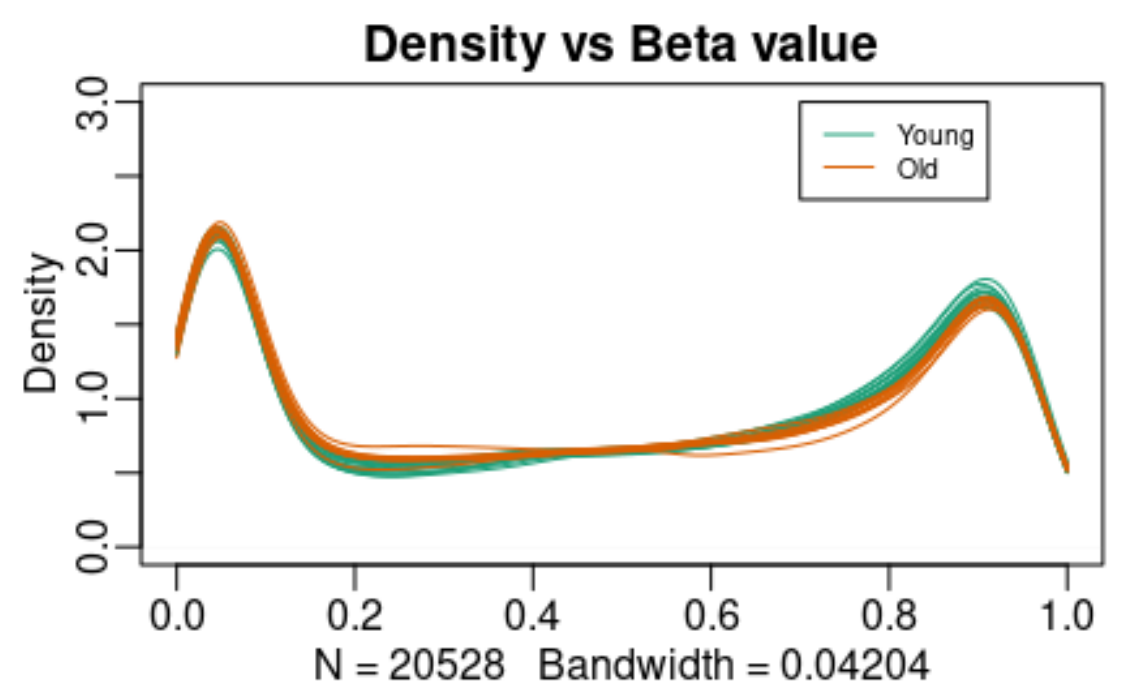

Supplemental Figure 1- Density distribution of $\beta$-values. - Density distribution of $\beta$ values across all the $22528 \mathrm{CpG}$ sites of the 22 samples. The orange lines represent the distribution of the young animals samples and the green lines represent the distribution of the old animal samples.

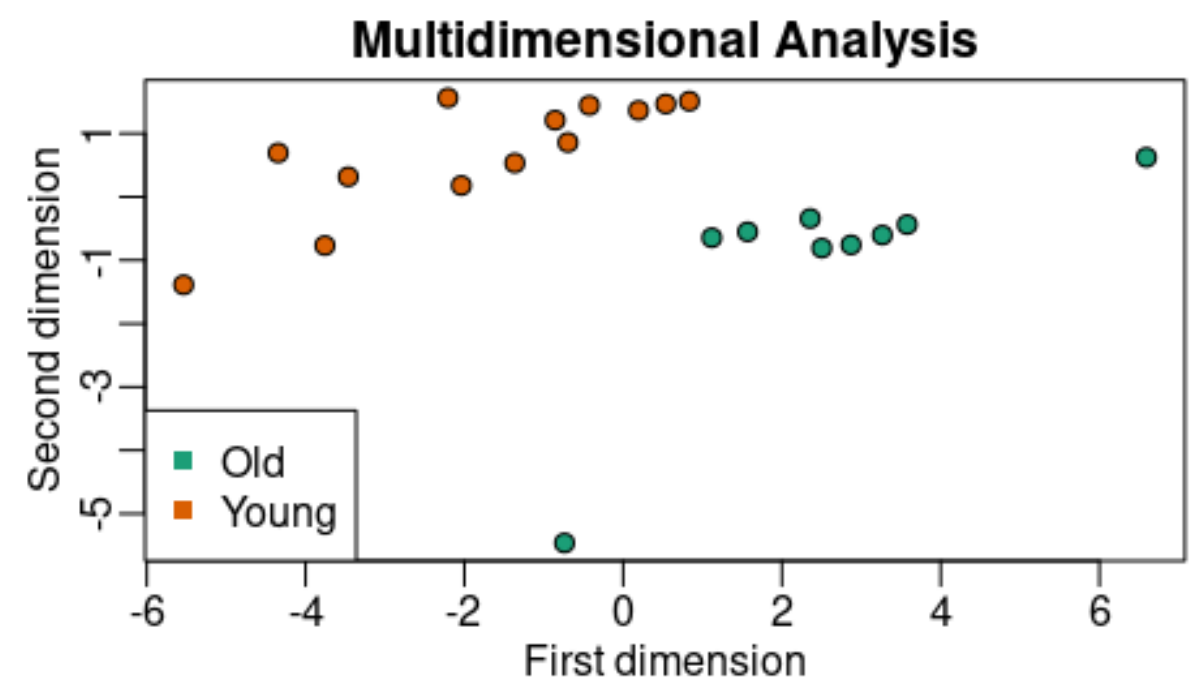

Supplemental Figure 2- Multidimensional scaling (MDS) plot of DNA methylation profiles in the hippocampus of young and old animals. This MDS plot of 20 samples shows that samples cluster according to age type, as expected. 


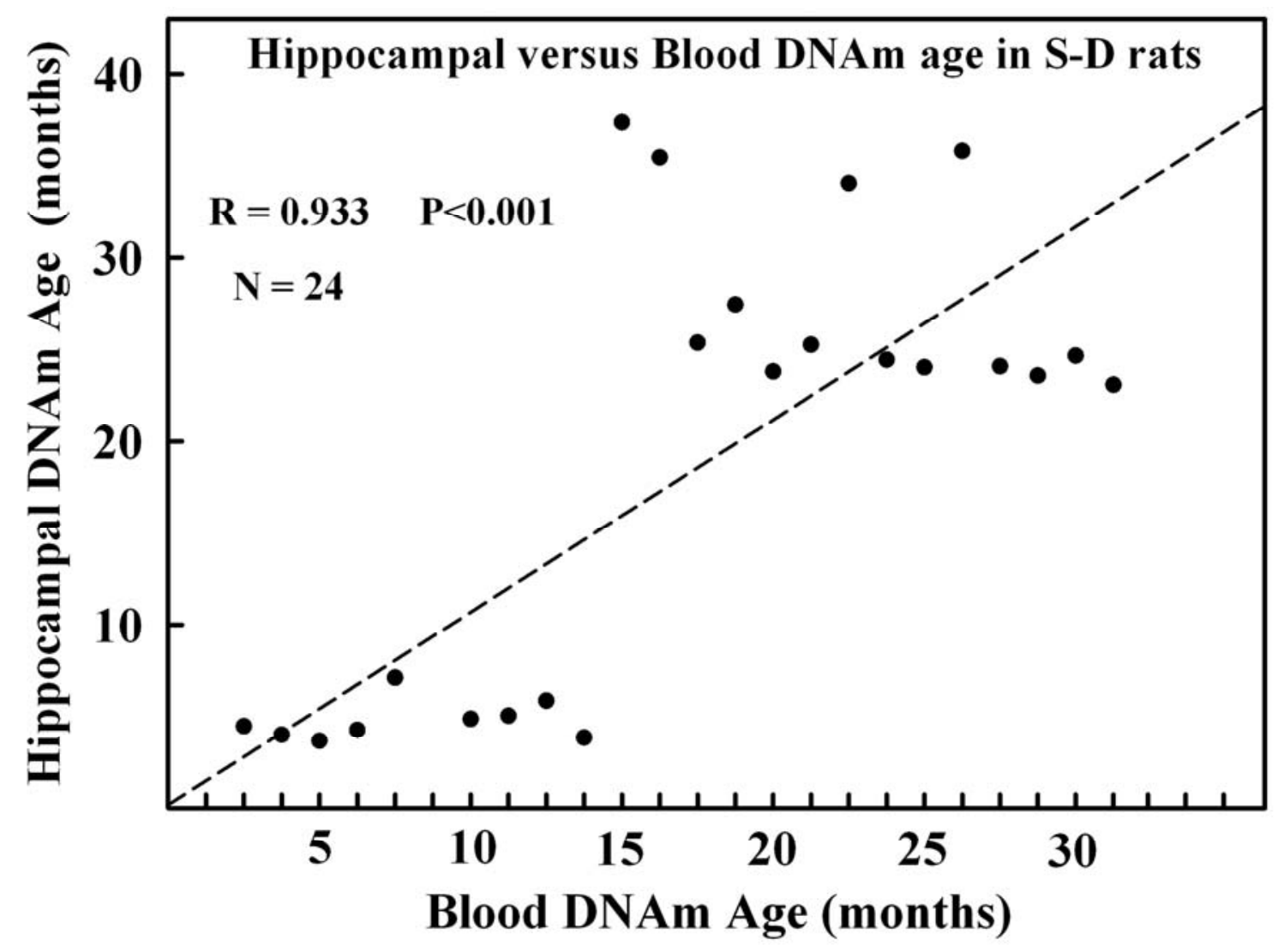

Supplemental Figure 3- Hippocampal versus blood DNAm age is rats. The regression, using training data, is highly significant which implies that in rats blood DNAm age is a reliable estimate of hippocampal DNAm age. 